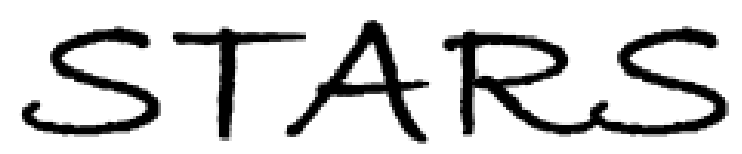

University of Central Florida

STARS

$1-1-2011$

\title{
Dispersion relation on the Kerr constant of a polymer-stabilized optically isotropic liquid crystal
}

\author{
Meizi Jiao \\ University of Central Florida \\ Jin Yan \\ University of Central Florida \\ Shin-Tson Wu \\ University of Central Florida
}

Find similar works at: https://stars.library.ucf.edu/facultybib2010 University of Central Florida Libraries http://library.ucf.edu

This Article is brought to you for free and open access by the Faculty Bibliography at STARS. It has been accepted for inclusion in Faculty Bibliography 2010 s by an authorized administrator of STARS. For more information, please contact STARS@ucf.edu.

\section{Recommended Citation}

Jiao, Meizi; Yan, Jin; and Wu, Shin-Tson, "Dispersion relation on the Kerr constant of a polymer-stabilized optically isotropic liquid crystal" (2011). Faculty Bibliography 2010s. 1435.

https://stars.library.ucf.edu/facultybib2010/1435

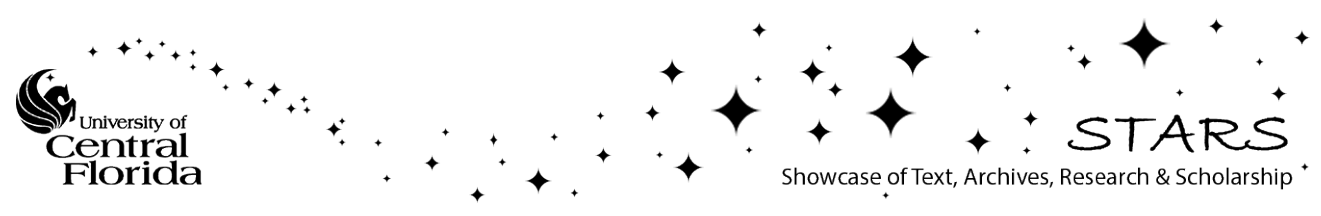




\title{
Dispersion relation on the Kerr constant of a polymer-stabilized optically isotropic liquid crystal
}

\author{
Meizi Jiao, Jin Yan, and Shin-Tson Wu \\ College of Optics and Photonics, University of Central Florida, Orlando, Florida 32816, USA
}

(Received 15 December 2010; published 11 April 2011)

\begin{abstract}
The dispersion relation on the Kerr constant $(K)$ of a polymer-stabilized isotropic phase (PSIP) liquid-crystal (LC) composite is investigated. Our experimental results show that $K$ decreases as the wavelength $(\lambda)$ increases. The single-band birefringence dispersion model is used to fit the $\lambda K$ values of the PSIP LC composite. Very good agreement between the experiment and physical model is obtained.
\end{abstract}

DOI: 10.1103/PhysRevE.83.041706

PACS number(s): 61.30.-v, 42.55.-f

\section{INTRODUCTION}

The Kerr effect is a second-order electro-optic (EO) effect [1]; it has been studied extensively in gases and liquids, such as benzene, $\mathrm{CS}_{2}, \mathrm{CCl}_{4}$, water, nitrotoluene, and nitrobenzene [2-4]. In a Kerr medium, the electric-field-induced birefringence is proportional to the electric field $(E)$ as

$$
\Delta n_{\text {ind }}=\lambda K E^{2},
$$

where $\lambda$ is the wavelength and $K$ is the Kerr constant [1]. Several high-speed $(\sim$ ns) EO modulators based on Kerr media have been demonstrated. However, the required operating voltage is quite high $(\sim \mathrm{kV})$ because the Kerr constant is fairly small $\left(\sim 10^{-12} \mathrm{~m} / \mathrm{V}^{2}\right)$.

Recently, polymer-stabilized blue-phase liquid crystals (PSBP LCs) have been developed [5-8]. The corresponding Kerr constant of these PSBP LC composites is $\sim 3-4$ orders of magnitude larger than that of isotropic liquids because of the short-range interaction of LC molecules. As a result, the operating voltage is significantly reduced $[9,10]$. PSBP exhibits some revolutionary features as compared to conventional nematic liquid crystals in the following aspects: (1) the isotropic dark state, which leads to wide and symmetric viewing angle, (2) a submillisecond gray-to-gray response time [11], which enables color sequential displays with tripled optical efficiency and resolution density, (3) no need for alignment layers, and (4) insensitivity to the cell gap, provided that in-plane switching (IPS) electrodes are employed. Therefore, PSBP liquid crystals are attractive for high-speed photonics and display applications [12,13]. In the PSBP precursor, the blue phase temperature range is widened $\left(7-9{ }^{\circ} \mathrm{C}\right)$ as compared to the pure blue phase range of the LC-chiral mixture $\left(1-2^{\circ} \mathrm{C}\right)$, but it is still relatively narrow. The UV curing process could take place either in a blue phase or in an isotropic phase to extend the temperature range. In the latter case, the LC composite is called the polymer-stabilized isotropic phase (PSIP). From a macroscopic viewpoint, both PSBP and PSIP LC composites are optically isotropic in the voltage-off state due to self-assembly nanosized lattice structures [14]. Therefore, the EO effects of PSBP and PSIP can be described by the Kerr effect.

However, Eq. (1) has raised some confusion: (1) Will the induced birefringence keep on increasing as the electric field increases? (2) Will the induced birefringence increase with the wavelength because $K$ is called a "constant"? The first confusion has been clarified recently, and an "extended Kerr effect" [15] has been developed to correctly predict the saturation phenomenon in the high-field region of a PSBP liquid crystal. Regarding the second question, some scattered experimental data and theories can be found in literature for isotropic liquids and gases [3,4], but, to the best of our knowledge, the implicit dispersion relation of the Kerr constant for PSIP has not been explored.

In this paper, we report the dispersion relation on the Kerr constant of a PSIP LC composite. Our experimental results show that the Kerr constant decreases gradually as the wavelength increases. We fit the measured Kerr constant with a single-band birefringence dispersion model $[16,17]$ and obtain excellent agreement. This information clearly answers the above-mentioned second possible confusion and, moreover, it is useful for designing a PSIP LC device for photonics and display applications by taking wavelength dispersion into consideration.

\section{EXPERIMENT}

Figure 1 shows the experimental setup for measuring the EO properties of a PSIP LC composite with different wavelengths. A narrowband interference color filter was placed in front of the white light source for selecting the wavelength. In our experiment, five color filters with transmission peaks at $\lambda \sim 450,500,550,633$, and $660 \mathrm{~nm}$ and bandwidths $\sim \pm 5 \mathrm{~nm}$ were used and their corresponding voltage-dependent transmittance $(V-T)$ curves measured. In our experiment, an ac voltage with 1-kHz square-wave frequency was applied. Since the white light source has an evident divergence angle, a group of lenses were used to collimate the light. In Fig. 1, we use one lens to represent this collimating lens group. A collimated light can travel a long distance without much divergence. Finally, a focusing lens was used to collect the transmitted light into a photodiode detector.

The PSIP LC used in this experiment comprised a $56 \mathrm{wt} \%$ nematic LC host MLC-14200 (Merck, $\Delta n=0.17, \Delta \varepsilon=30$, clearing point $\left.T_{c}=94^{\circ} \mathrm{C}\right), 29 \mathrm{wt} \%$ chiral dopants ( $24 \%$ CB15 and $5 \%$ ZLI-4572, Merck), and $15 \mathrm{wt} \%$ monomers (6\% RM257 and 9\% EHA). The LC-monomer mixture was injected into an IPS cell with an electrode width $\sim 10 \mu \mathrm{m}$, electrode gap $\sim 10 \mu \mathrm{m}$, and cell gap $\sim 7.5 \mu \mathrm{m}$. The cell was cured by an UV lamp $(\lambda \sim 365 \mathrm{~nm})$ at $70{ }^{\circ} \mathrm{C}$, which was well above the isotropic temperature of the LC-monomer mixture. The LC cell was sandwiched between two crossed polarizers with the striped electrodes oriented at $45^{\circ}$ with respect to the polarizer's transmission axis. We measured the $V$ - $T$ curves at room temperature $\left(\sim 23^{\circ} \mathrm{C}\right)$ under different wavelengths. 


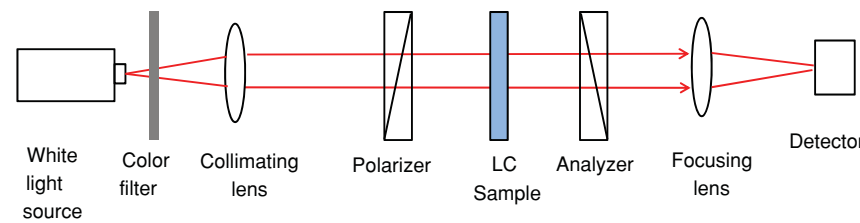

FIG. 1. (Color online) Experimental setup for measuring the $V-T$ curves of a polymer-stabilized isotropic LC composite at different wavelengths.

Figure 2 shows the measured $V$ - $T$ curves of the LC composite at five wavelengths. From left to right, the corresponding wavelengths are $450,500,550,633$, and $660 \mathrm{~nm}$. Because the luminous flux of each wavelength is different, we normalize the transmittance of each wavelength to its own peak value. The operating voltage is lower at a shorter wavelength for two reasons: (1) The induced phase retardation is inversely proportional to the wavelength, thus a shorter wavelength will exhibit a larger phase retardation under the same driving voltage; and (2) under normal dispersion conditions the induced birefringence decreases as the wavelength increases. Considering these two factors, the on-state voltage is lower for a shorter wavelength.

\section{PHYSICAL MODELS AND DISCUSSIONS}

To study the detailed dispersion relation of the PSIP LC composite, we carried out simulations to fit the experimental data shown in Fig. 2. First, we calculated the electric-field distribution in our IPS cell at voltage-on states using a finiteelement method [18]. Then for each wavelength we used the following extended Kerr effect model $[15,19]$ to calculate the induced birefringence based on the electric-field distribution:

$$
\Delta n=\Delta n_{\text {sat }}\left\{1-\exp \left[-\left(\frac{E}{E_{s}}\right)^{2}\right]\right\},
$$

where $\Delta n_{\text {sat }}$ is the saturated induced birefringence and $E_{s}$ is the saturation electric field. Afterward, we used the extended Jones

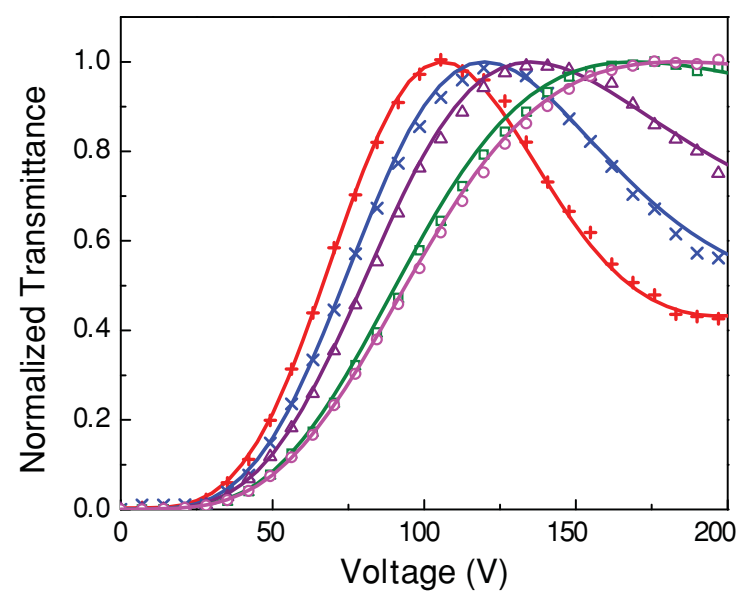

FIG. 2. (Color online) Measured (dots) and fitted (lines) $V-T$ curves of the PSIP LC cell at different wavelengths. From left to right, the corresponding wavelengths are $450,500,550,633$, and $660 \mathrm{~nm}$
TABLE I. Fitting results of the saturated birefringence $\left(\Delta n_{\text {sat }}\right)$, saturation electric field $\left(E_{s}\right)$, and Kerr constant of the PSIP LC composite at the corresponding wavelengths.

\begin{tabular}{lccc}
\hline \hline$\lambda(\mathrm{nm})$ & $\Delta n_{\text {sat }}$ & $E_{s}(\mathrm{~V} / \mu \mathrm{m})$ & $K\left(\mathrm{~nm} / \mathrm{V}^{2}\right)$ \\
\hline 450 & 0.0730 & 6.8 & 3.51 \\
500 & 0.0695 & 6.8 & 3.01 \\
550 & 0.0670 & 6.8 & 2.63 \\
633 & 0.0635 & 6.8 & 2.17 \\
660 & 0.0630 & 6.8 & 2.06 \\
\hline \hline
\end{tabular}

matrix method $[20,21]$ to calculate the averaged transmittance. The extended Kerr effect model [Eq. (2)] clearly explains the convergence phenomenon observed in the high-field region. The fitting results with Eq. (2) are shown in Fig. 2, in which dots represent experimental data and solid lines are fitting curves. They overlap with each other very well at each wavelength. There are two fitting parameters, $\Delta n_{\text {sat }}$ and $E_{s}$. The fitting results at each wavelength are listed in Table I.

It is interesting to note that the fitting results of $E_{s}$ are the same for all the wavelengths studied. Because at a given temperature and voltage the LC director reorientation profile is determined by the balanced elastic and electric torques, the LC director distribution will not be affected by the wavelength. However, the $V-T$ curves will be different for different wavelengths because the induced birefringence $\left(\Delta n_{\text {sat }}\right)$ does depend on the wavelength, as shown in Table I. Moreover, the phase retardation varies with the wavelength.

The lower part of Fig. 3 shows the wavelength-dependent $\Delta n_{\text {sat }}$ for the PSIP LC composite; here squares stand for the experimental data listed in Table I and the solid line represents the fitting curve with the following single-band birefringence dispersion model $[15,16]$ :

$$
\Delta n_{\mathrm{sat}}=G \frac{\lambda^{2} \lambda^{* 2}}{\lambda^{2}-\lambda^{* 2}} \stackrel{\lambda \gg \lambda^{*}}{\longrightarrow} G \lambda^{* 2}
$$

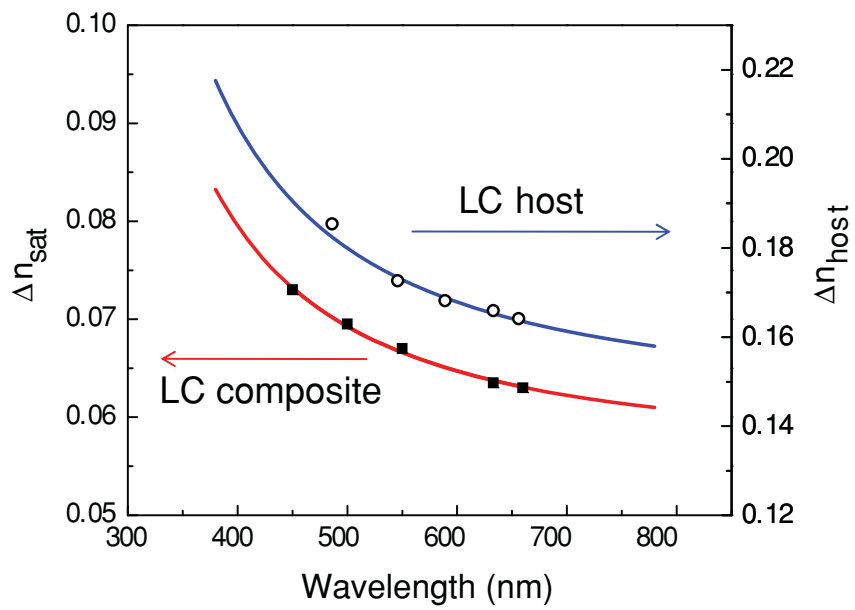

FIG. 3. (Color online) Dispersion relations of the saturated induced birefringence of the PSIP LC composite (filled squares) and its nematic LC host MLC-14200 (open circles). Solid lines are fitting curves. 
where $\lambda^{*}$ is the average resonant wavelength of the LC composite and $G$ is a proportionality constant. For a conjugated LC compound, the two $\pi \rightarrow \pi^{*}$ electronic transitions usually occur in the UV region [22] and $G$ decreases as the temperature increases according to the order parameter $S=\left(1-T / T_{c}\right)^{\beta}$; here the exponent $\beta$ is a material constant [23]. For a multicomponent LC mixture, there will be several different $\pi \rightarrow \pi^{*}$ electronic transition wavelengths, depending on the conjugation length of the individual compound. In the off-resonance region, the single-band model treats these $\pi \rightarrow \pi^{*}$ transition wavelengths as an average wavelength $\lambda^{*}$. This model works equally well for both single-compound and eutectic mixtures [24]. Similar to nematic LCs, the dispersion of the PSIP LC composite also follows the single-band birefringence dispersion model well. Through fittings, we find $\lambda^{*} \sim 216 \mathrm{~nm}$ and $G \sim 1.205 \times 10^{-6} \mathrm{~nm}^{-2}$.

To correlate the dispersion property of the LC composite with the LC host, we also measured the birefringence dispersion of the LC host (MLC-14200) using an Abbe refractometer. The measured data are included in the upper part of Fig. 3, where the solid (blue) line is the fitting curve based on Eq. (3) with $\lambda^{*} \sim 218 \mathrm{~nm}$ and $G \sim 3.047 \times 10^{-6} \mathrm{~nm}^{-2}$. The two obtained $\lambda^{*}$ values agree with each other to within $1 \%$. This is not surprising because the dispersion of the PSIP LC composite is primarily determined by the LC host employed. On the other hand, the decreased $G$ value of the polymer-stabilized optically isotropic LC composite mainly originates from the decreased LC concentration. In our PSIP LC composite, the LC concentration is only $\sim 56 \mathrm{wt} \%$ and the rest are chiral dopants and photocurable monomers. Thus, the expected decrease in birefringence is $\sim 44 \%$, but the obtained $G$ value is decreased by $\sim 60 \%$. This excessive $\sim 16 \%$ reduction could be attributed to two unaccounted factors: (1) The LC composite has a lower clearing temperature than its host, and (2) some LC molecules could be trapped by the polymer networks during polymerization process. The lower clearing point leads to a decreased order parameter while the trapped LC molecules are hardly reoriented by the applied electric field.

From Fig. 3, the $\Delta n_{\text {sat }}$ of the PSIP LC composite decreases as the wavelength increases. When the wavelength is much longer than the resonant wavelength, i.e., $\lambda \gg \lambda^{*}$, the induced birefringence gradually saturates to a plateau $\left(G \lambda^{* 2}\right)$ according to Eq. (3). When a PSIP LC composite is used in the infrared region (say, $\lambda>1 \mu \mathrm{m}$ ), the induced birefringence will reach a saturation level, similar to nematic liquid crystals.

In the low electric-field region $\left(E \ll E_{s}\right)$, the extended Kerr effect [Eq. (2)] is reduced to conventional Kerr effect [Eq. (1)]. With Taylor's expansion, the Kerr constant is expressed as [15]

$$
K=\Delta n_{\text {sat }} / \lambda E_{s}^{2} .
$$

As shown in Table I, the obtained Kerr constant decreases gradually with wavelength. Based on Eq. (4), we derive the dispersion relation of Kerr constant as follows:

$$
K=\frac{G \lambda \lambda^{* 2}}{E_{s}^{2}\left(\lambda^{2}-\lambda^{* 2}\right)}=\frac{A \lambda \lambda^{* 2}}{\lambda^{2}-\lambda^{* 2}},
$$

where $A=G / E_{s}^{2}$ is a proportionality constant. In Fig. 4, we fitted the Kerr constant data with Eq. (5) using $\lambda^{*} \sim 216 \mathrm{~nm}$

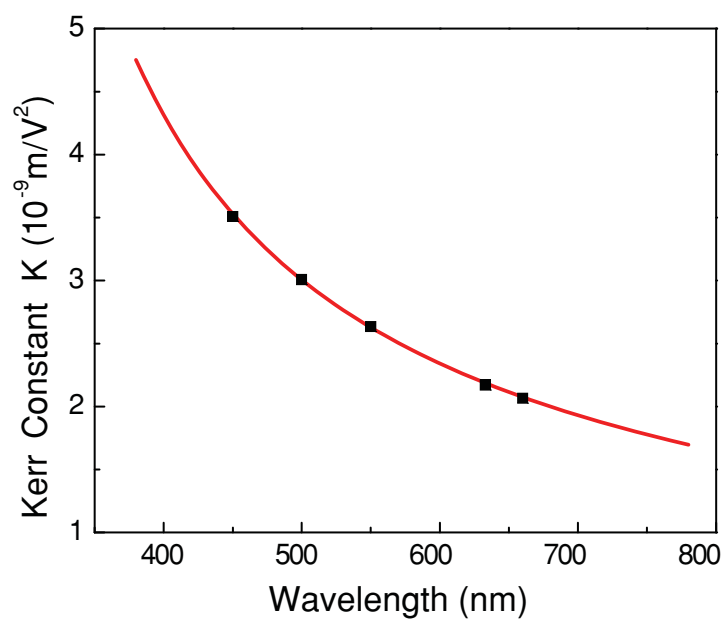

FIG. 4. (Color online) Measured Kerr constant (squares) and the fitting results (solid line) of the PSIP LC composite at different wavelengths.

and $A \sim 0.0262 \mathrm{~nm}^{-1}$. The well-fitted data clearly demonstrate the implicit wavelength dependency of the Kerr constant.

In the low-field region, the conventional Kerr effect is valid and $\lambda K$ is a coefficient for calculating the induced birefringence under a given electric field. From Eqs. (3) and (4), the term $\lambda K$ has the same dispersion relation as $\Delta n_{\text {sat }}$, which can be described well by the single-band model. Therefore, to take the implicit wavelength dependency of the Kerr constant into consideration, it would be clearer to rewrite the conventional Kerr equation as

$$
\Delta n_{\text {ind }}=K_{o}(\lambda, T) E^{2},
$$

where $K_{o}=\lambda K$ (in units of $\mathrm{m}^{2} / \mathrm{V}^{2}$ ) is the modified Kerr coefficient representing the Kerr effect at a given wavelength and temperature. Apparently, $K_{o}$ decreases with the wavelength and temperature according to the single-band model. The temperature-dependent Kerr constant $K$ is indeed following the order parameter, as validated experimentally [25]. In the high-field region, the conventional Kerr effect is no longer valid so that the extended Kerr effect [Eq. (2)] has to be used.

\section{CONCLUSIONS}

The dispersion relation of a polymer-stabilized optically isotropic LC composite is investigated. The experimental results of $\lambda K$ follow the same dispersion trend as its nematic host LC, and can be described well by the single-band birefringence dispersion model. For display and photonic applications, the wavelength-dependent Kerr constant has to be taken into consideration.

\section{ACKNOWLEDGMENT}

The authors are indebted to the Industrial Technology Research Institute (Taiwan) for financial support. 
[1] J. Kerr, Philos. Mag. 50, 337 (1875).

[2] T. H. Havelock, Phys. Rev. 28, 136 (1909).

[3] J. W. Beams, Rev. Mod. Phys. 4, 133 (1932).

[4] A. Yariv and P. Yeh, Optical Waves in Crystal: Propagation and Control of Laser Retardation (Wiley, New York, 2002).

[5] H. Kikuchi, M. Yokota, Y. Hisakado, H. Yang, and T. Kajiyama, Nat. Mater. 1, 64 (2002).

[6] Y. Haseba, H. Kikuchi, T. Nagamura, and T. Kajiyama, Adv. Mater. 17, 2311 (2005).

[7] Z. Ge, S. Gauza, M. Jiao, H. Xianyu, and S. T. Wu, Appl. Phys. Lett. 94, 101104 (2009).

[8] L. Rao, J. Yan, S. T. Wu, S. Yamamoto, and Y. Haseba, Appl. Phys. Lett. 98, 081109 (2011).

[9] L. Rao, Z. Ge, S. T. Wu, and S. H. Lee, Appl. Phys. Lett. 95, 231101 (2009).

[10] M. Jiao, Y. Li, and S. T. Wu, Appl. Phys. Lett. 96, 011102 (2010).

[11] K. M. Chen, S. Gauza, H. Xianyu, and S. T. Wu, J. Disp. Technol. 6, 49 (2010).

[12] Y. H. Lin, H. S. Chen, H. C. Lin, Y. S. Tsou, H. K. Hsu, and W. Y. Li, Appl. Phys. Lett. 96, 113505 (2010).
[13] H. Y. Liu, C. T. Wang, C. Y. Hsu, T. H. Lin, and J. H. Liu, Appl. Phys. Lett. 96, 121103 (2010).

[14] S. Meiboom, J. P. Sethna, P. W. Anderson, and W. F. Brinkman, Phys. Rev. Lett. 46, 1216 (1981).

[15] J. Yan, H. C. Cheng, S. Gauza, Y. Li, M. Jiao, L. Rao, and S. T. Wu, Appl. Phys. Lett. 96, 071105 (2010).

[16] S. T. Wu, Phys. Rev. A 33, 1270 (1986).

[17] S. T. Wu, C. S. Wu, M. Warenghem, and M. Ismaili, Opt. Eng. 32, 1775 (1993).

[18] J. Jin, The Finite Element Method in Electromagnetics, 2nd ed. (Wiley-IEEE, New York, 2002).

[19] J. Yan, M. Jiao, L. Rao, and S. T. Wu, Opt. Express 18, 11450 (2010).

[20] A. Lien, Appl. Phys. Lett. 57, 2767 (1990).

[21] Z. Ge, X. Zhu, T. X. Wu, and S. T. Wu, J. Opt. Soc. Am. A 22, 966 (2005).

[22] S. T. Wu, E. Ramos, and U. Finkenzeller, J. Appl. Phys. 68, 78 (1990).

[23] I. Haller, Prog. Solid State Chem. 10, 103 (1975).

[24] J. Li and S. T. Wu, J. Appl. Phys. 96, 170 (2004).

[25] L. Rao, J. Yan, and S. T. Wu, J. Soc. Inf. Disp. 18, 954 (2010). 